\title{
MULTIMEDIA AND THE LEARNER'S EXPERIENCE OF NARRATIVE DIANA LAURILLARD
}

\section{Introduction}

This paper reports on research findings which show that the narrative structure of multimedia programs, or sometimes the lack of it, affects learners' comprehension, often adversely $[1,2]$. It also reports on initial findings from our current research which aims to develop a theoretical understanding of the forms and functions of narrative in interactive media, based on empirical research, and capable of informing instructional design.

\section{A Framework for Analysing Educational Media}

In a recent book, I have suggested that the various educational media (print, audio, video, computers) can be compared and contrasted for the contribution they each make to supporting the different aspects of the teaching-learning process [3]. The analysis presented there, which characterised the process as a 'conversational framework' was related to the work of Gordon Pask. The argument begins with a description of the teaching-learning process as consisting essentially of:

- discussion between teacher and learner at the level of descriptions

- interaction between the learner and some special aspect of the world defined by the teacher

- adaptation of that special world by teacher, and of action by learner

- reflection on learner's performance by teacher and learner.

With this, it is possible to consider the extent to which each medium can support each part of the process. For example, print can do little more than convey the teacher's description, i.e. just one side of the discussion. The text may request that the learner express their own description, but the medium cannot require it in the way that a face-to-face dialogue can. It can express the teacher's reaction to a possible learner description, but cannot react to an actual expression of the learner's understanding in the way a face-to-face dialogue can. On the other hand, a medium such as computer-mediated conferencing can support the two-way dialogue very well, albeit in a more attenuated way than the face-to-face version. Unlike print, however, which can use diagrams and pictures, it usually has only text as the medium of expression for the teacher.

Interaction between the learner and the world is a vital part of the learning process because it is this that situates academic knowledge in our experience of the world. If we are to understand the world, whether it is a child learning arithmetic, or a student learning about economics, if our academic knowledge remains abstract and formal without any meaningful interpretation, then it is useless: it does not enable more effective action in the world, which is the primary value of academic knowledge. The world the learner interacts with is necessarily a teacher-constructed part of the world - a classroom experiment, a field-trip, a poem, etc., depending on the subject matter. It is the teacher's task to construct the conditions of the learner's interaction such that their experience enables them to learn - the experiment demonstrates that hot air rises, or the field trip shows what the topography of a fault line looks like, or reading the poem out loud shows how the metre works. On the basis of that particular experience the teacher can then begin to build general, abstracted descriptions. The interactive media, i.e. computer-based media, can support the learner in what is otherwise only possible through real-world experience. 
Adaptation and reflection connect the discussion with the interaction. The teacher reflects on the learner's performance and adapts what they say accordingly, as a way of making their teaching responsive to the learner's needs. The student uses what the teacher says to adapt their actions, and reflects on the result in order to modify and develop their own idea until it accords with the teacher's, in the process of discussion. The learning process is completed insofar as teacher and learner agree on a description, e.g. an explanation of weather conditions, an interpretation of geological strata, an analysis of a poem. Few media can support the learner in either adaptation or reflection.

With this as a framework, we can see that different media contribute to the learning process in very different ways. Video is good at conveying the teacher's ideas, through words, pictures and events, but can do nothing, itself, to enable the learner to express their own ideas. Traditional computer-assisted learning, with its emphasis on text and diagrams, is a poor medium for expressing the teacher's description, but it remains an interactive medium that can model some aspects of the world very well, and can provide the student with a simulated environment with which to interact.

Advances in the technological media now provide interactive access to large text and audiovisual databases, and make it possible for learners to conduct on-line searches through attractive information. This has brought a flood of new recruits to the rhetoric of 'learner-oriented', 'constructivist', 'exploratory' learning. This is what these multimedia databases with their hypertext access appear to offer, so that is what is now proclaimed for the technology. However, educators who hold dear those ideas must decide if this is a valid claim. To what extent does multimedia of this kind really support exploratory learning?

\section{The Role of Narrative in Comprehension}

There is an important contrast between traditional media and teaching methods and the more learner-controlled approaches: the traditional methods of lectures, print, video, audio are 'narrative' media, that require a storyline, that put the teacher in the role of 'storyteller' and the learner in the role of 'listener'. This is a venerable tradition in education, and one to be appreciated as often being an impressively efficient and effective way of preserving and transmitting our cultures? knowledge. Narrative structure is fundamental to comprehension [4] to the extent that when it is clearly absent from certain forms of multimedia, this can seriously undermine comprehension of the material $[1,2,5]$.

By contrast with traditional media, one of the key benefits for interactive media is seen as being the lack of imposed structure, giving much greater freedom of control to the user. However, in the context of instruction, this benefit runs counter to the learner's need to discern structure if there is a message to be understood. We have found, from observation in previous research studies, that learners working on interactive media with no clear narrative structure display learning behaviour that is generally unfocused and inconclusive. Only when teachers prepare instructional worksheets, or offer supervision, thus imposing some form of organising structure, is learners' work likely to be productive $[1,6]$. Thus one of the key benefits of interactive media, the greater learner control it offers, becomes pedagogically disadvantageous if it results in mere absence of structure.

Narrative structure is one of the most important ways in which the instructional message comes to be understood, and we have to learn how to manage a medium that undermines its power if it is to succeed in the educational context. 


\section{Narrative in Multimedia}

It is typical of a narrative medium, whether print or video, that the denouement is not fully revealed at the beginning - only the general direction - so that the author can build towards it. It is also necessarily the case that the authorial voice dictates the route to the goal, and decides when the goal has been reached; the reader/listener can only follow, learning through 'acquisition' what is offered. What happens to the learning experience when the same teaching is transferred to a multimedia format?

To clarify the nature of the changes that must occur, it will be useful to illustrate them with a particular example, taken from a current project at the Open University. It involves part of a course on 'Homer: Poetry and Society', which looks at the nature of the relationship between the Homeric poems and the archaeological data on Ancient Greece. The course is taught currently through print (poems, commentaries, essays, study guide), video (of archaeological sites, site plans, excavations, material objects) and audio (readings of poetry, expert discussions). In the format of these narrative media, a section of the teaching will have quite a complex structure, with different media (printed study guide, video of archaeological site, students' notes, published text) carrying different parts of the storyline, for example:

- hypothetical question Guide

- critique of assumption Guide

- presentation of evidence Video

- elicited student's response Notes

- interpretation of evidence Guide

- contradictory evidence Text

- interpretation of evidence Guide

- $\quad$ synthesis at higher level Guide

The objective is to enable the student to understand the concluding synthesis and the reasons for it as a balanced position.

This material can be transferred to interactive multimedia in the form of a multiply-linked audiovisual database, where sections of the poems are linked to commentaries, video sequences, and pictures of archaeological artefacts; place names are linked to photos, site plans, a video walkthrough, etc. It makes an impressive resource for the scholar-researcher (similar to the 'Perseus' disc). The student as researcher may not always work so effectively with such a resource, however. Without any narrative structure, how does the novice cope?

This defines the framework for our current research project, designed to investigate how narrative and non-narrative structures function in interactive media, and to what extent existing structures help learners understand the material. The research is using material such as the Homer disc and its print original in the empirical studies.

\section{The Research Question}

The project is called 'MENO: Multimedia, Education and Narrative Organisation'. One focus of the work is to discover the means by which the learner might find their own way in the world of knowledge, i.e. might really engage in exploratory learning. The Socratic dialogues are 
frequently offered as the ideal form of teaching - through careful questioning the teacher elicits the knowledge, guiding the learner through the gradual revelation of what is to be known. It is still regarded as the epitome of good teaching. There is a sense, however, in which this form of teaching is of no help at all to the learner. The expertise is all in the conduct of the questioning, the contributions from the slave being little more than 'yes' or 'no' answers to Socrates' questions, and without that guidance, the learner is lost, unable to reproduce the sequence that reveals what is to be known. If Meno's slave had been able to arrive at Socrates' truth unaided by Socrates' questions, framing his own questions instead, that would really have been a demonstration that he already had the knowledge. But that is not possible for the learner. Meno's paradox stands: how can you learn something you know nothing of? And that is the paradox that greets the student who comes unaided to a multimedia database.

One of the outcomes that we hope to achieve from our research study, therefore, is a clarification of the approach Meno's slave should have taken, faced with a resource like Socrates. When learners encounter the extensive resource of a multimedia database, how are they to manage the process of discovery, of understanding, of acquisition of the salient knowledge in a meaningful way? Is there a principled dialogue, or investigative technique, the nature of which could be made available in the interactive format of such resources?

\section{The Design of the Homer Disc}

Consider the question in relation to the course on Homer, described above. Clearly, given the complex narrative line that the academics wanted to present, it was unlikely that students would attain the same objectives if left to explore this extensive resource unaided. It was important that the design of the disc containing all this material should allow the student to browse and navigate at will, but it was even more important to structure the material in such a way that the original objectives for the course could still be attained. However, it would be a serious misuse of the medium to simply conduct students through the narrative line of argument outlined above, as was appropriate for the print/video/audio version. Multimedia must be an active medium for the user, creating opportunities for the learner to make decisions, formulate and test hypotheses, make choices, construct interpretations, etc. But if the learners do not yet know the story, how are they to construct the activities that will take them in the direction intended by the teacher? We seemed to be thwarted by Meno's paradox. The solution was to make the narrative figural, enabling the learner to see its structure and hence be able to participate in its elaboration. We had to turn the story inside out. Instead of slowly revealing the twists and turns of the plot, the evidence and counter-evidence, reinterpretation, critique, synthesis, the learner had to be presented with all this in outline, being aware of the form of the final denouement if not its detailed content, so that they could then become party to the decisions on how to get there. This was the first major design decision: to expose the narrative line as a series of goals defining the investigations of the material to be carried out. This was done by analysing the printed study guide and the script of the video to discern the key component points building to the main conclusion within each unit, i.e. within each week's work. Figure 1 shows an example of the set of investigation activities for Week 1, generated from the printed text, and defining for the student the shape and direction of their work, without undermining their freedom of exploration, still available if they wished via the indexes to the resources shown at the top of the screen. 


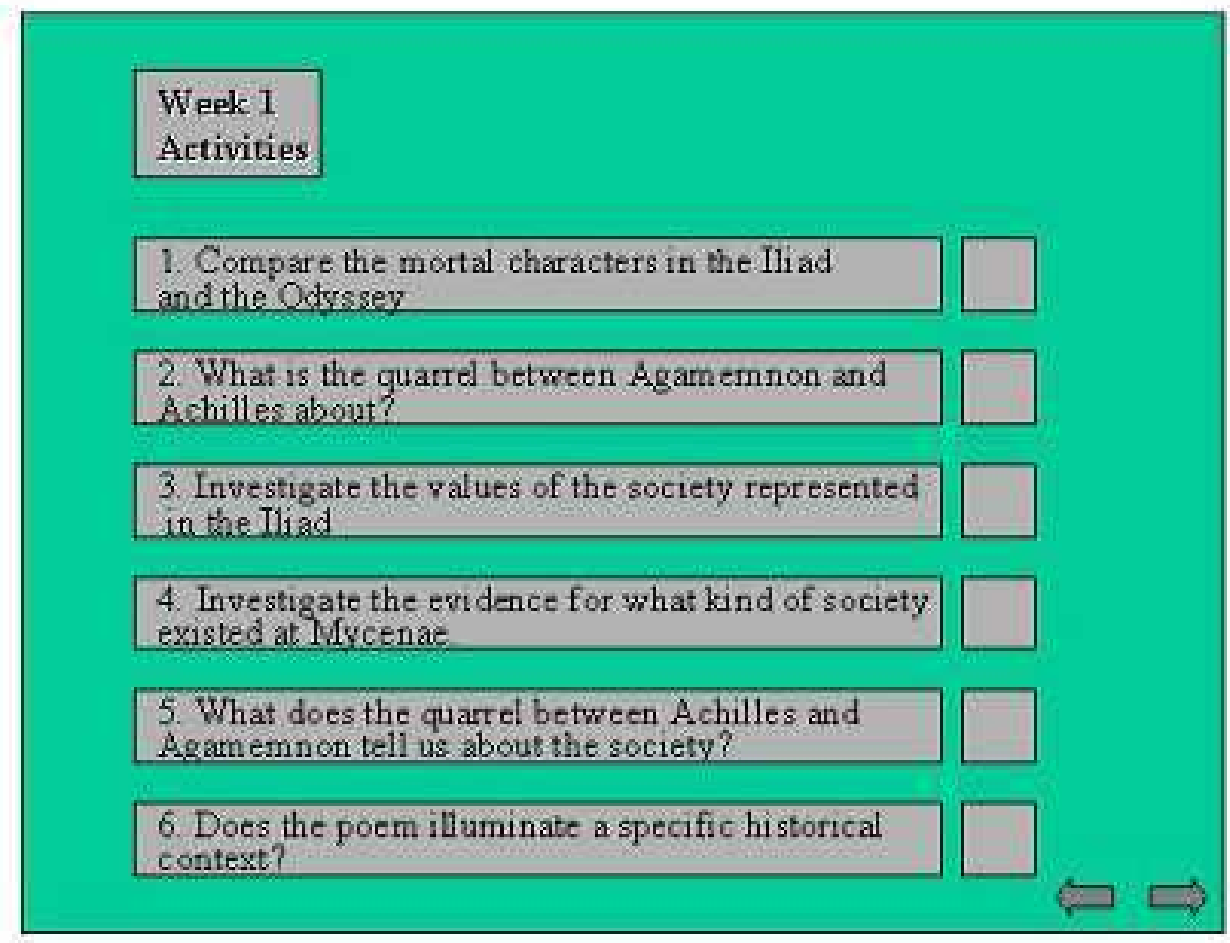

Figure 1: An example of the set of investigation activities for a week's work.

Once the investigation is selected, the student then still needs some support in conducting it.Each one offers a set of guidelines, as shown in Figure 2.

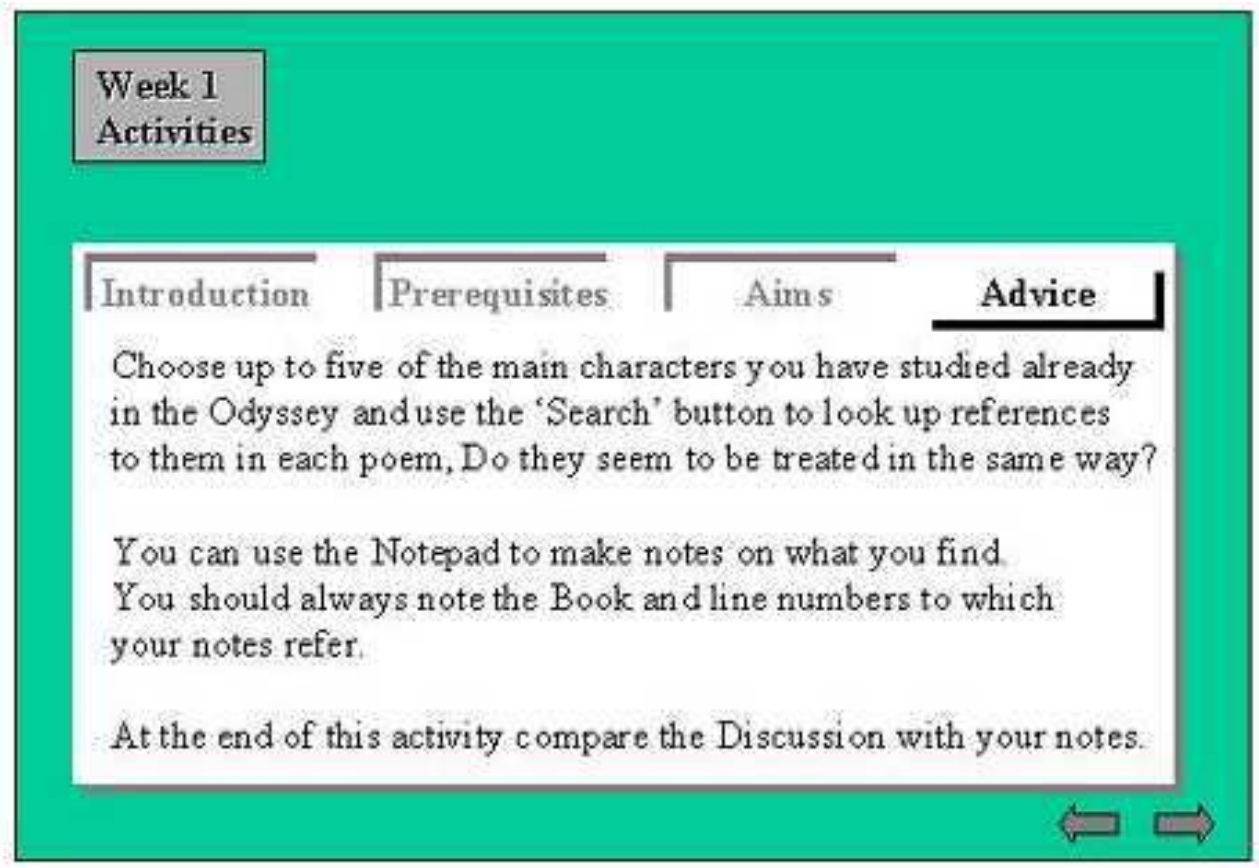

Figure 2: Advice offered to students for Activity 1. 
The introduction to the question outlines what it means, and why it is important. The prerequisites suggest what they should already have covered in print, audio or video in order to attempt the question. It would be difficult for students to make an appropriate judgement about this before they have become familiar with the resources, and with what the question entails. The aim defines the amount of work they should expect to carry out - in this case, for example, how many characters to study, how many references for each - again because it is difficult for students to know the comparative priority to attach to each question. The advice suggests how they might start the investigation, suggests good practice hints, or pre-empts some possible unsuitable approaches, depending on the question. Figure 2 shows the advice offered for Activity 1.

With this level of support for the activities they are to carry out, the learners can expect to be aware of why they are doing each investigation, and what part it plays overall. If the task is meaningful then they are better able to make their own contribution to it, or to take control and deviate from the suggested path if they wish.

The next major design feature was to ensure that the program made the learner as active as possible, able to set their own tasks within the given tasks, and to carry through their own analysis in relation to the question. Within the activity specified, the student now has the freedom to explore the texts and other resources as much as they wish in order to build up the evidence they need. The example in Figure 2 is an essentially poem-related question, so they will confine their search here to the poems, using the search facility supplied by the Toolbook software. Figure 3 shows part of the result of the search for any of the five heroes who appear in both poems.

\begin{tabular}{|c|c|c|}
\hline \multicolumn{3}{|c|}{$\begin{array}{l}\text { The lliad of Hom er } \\
\text { BOOK TWO }\end{array}$} \\
\hline \multicolumn{3}{|c|}{$\begin{array}{l}20 \text { Dream stood then beside his head in the likeness of Nestor, } \\
\text { Neleus' son, whom Agamemnon honoured beyond all } \\
\text { elders beside. In Nestor's likeness the divine Dream spoke to } \\
\text { 'Son of wise Atreus breaker of horses, are you sleeping? } \\
\text { He should not sleep night long who is a man burdened with } \\
25 \text { and re sponsibility for a people and cares so numerous. } \\
\text { Listen quickly to what I say, since I am a messenger } \\
\text { of Zeus, who far away cares much or you and is pitiful. } \\
\text { Zeus bids you arm the flowing-haired Achaians for battle } \\
\text { in all haste; since now you might take the wide-wayed city }\end{array}$} \\
\hline \multicolumn{2}{|c|}{ Search results } & Notepad \\
\hline \multirow{4}{*}{\multicolumn{2}{|c|}{$\begin{array}{l}\text { Iliad Glossary } \\
\text { Iliad Book Eleven } \\
\text { Iliad Book Eight } \\
\text { Iliad Book Fourteen }\end{array}$}} & Searched for Westor in the Iliad \\
\hline & & Book $2, \ln 20$ \\
\hline & & Nestor is a respected elder. \\
\hline & & \\
\hline \multicolumn{3}{|c|}{ Iliad Book Two } \\
\hline \multicolumn{2}{|c|}{ Tliad Book Sewen } & \\
\hline
\end{tabular}

Figure 3: The result of a search conducted for Activity 1

The Reminder displays the length of time the learner is expected to spend on this Activity. The Notepad automatically records the name of the Activity, so that the learner is always aware of what issue they are investigating at any one time. It also records the search that was carried out. 
The text window shows the first occurrence of the search items in the text of Book One of the Iliad, showing also the hypertext nodes available, underlined. The remainder of the Notepad is available for the student to take notes on what they have found from the search. They are advised to record the location in the text because Toolbook cannot do this for them, an annoying feature of the software which nonetheless inculcates in the learner the good habit of recording the source of a reference. The Notepad can record extensive notes, which can then be printed out, or edited, at the end of the session for use when the time comes to write up an essay for an assignment.

Having collected some evidence and recorded their notes and interpretations, the learner now needs to achieve closure on the task. They may have spent the allotted time and covered the requisite number of searches, but how are they to know if they have achieved what was intended? Providing feedback on work carried out is very difficult when the exercise is as openended as these tend to be. However, it is possible to give the student some sense of how close they have come to the objective by giving them access to a model answer. Once they have made some kind of input to the Notepad, they can call up the 'Discussion' associated with that Activity, as shown in Figure 4 for Activity 1.

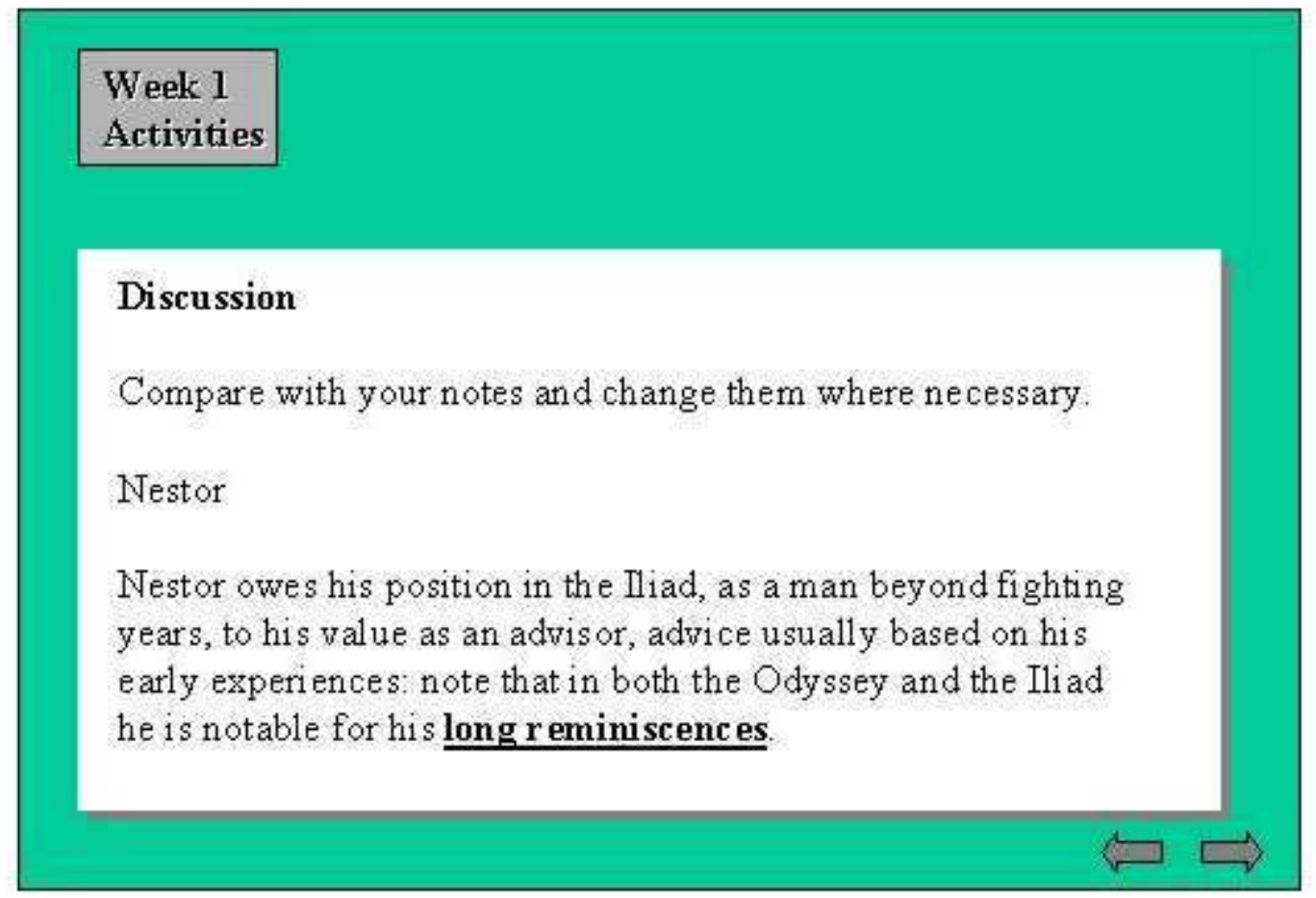

Figure 4: The expert's 'Discussion' associated with Activity 1.

The discussion is pre-designed text for each Activity, giving the academic's analysis of the issue, similar to that originally provided in the printed study guide, but with the important difference that instead of following on immediately from the presentation of the issue, the learner has in this case been required to do some investigation and analysis of their own before gaining access to the academic's interpretation. It was not clear that this feature would necessarily be welcomed by students, but initial evaluation with students who had studied the traditional course the previous year suggested that they very much appreciated being forced, or perhaps enabled, to consider and develop their own analysis first, before seeing what the expert had written. In some cases, they would use the Discussion to return to the Notepad and elaborate their analysis. In others, they found from the comparison that they had contributed something of their own, not found in the expert's analysis. This is a very important feature of the delayed feedback made possible by the 
interactive media, because it encourages students to develop their own ideas and begin to build up confidence that they do have something of their own to contribute.

A different kind of activity might require students to consult both text and other resources on the disc. The fourth Activity in Week One, for example, 'Investigate the evidence for what kind of society existed at Mycenae' calls on the archaeological evidence as well. A text search on words such as 'soldiers', 'king', 'home', for example, might lead to clues in the text about the natures of the society and its values. Further evidence for this Activity might be sought from the archaeological site maps on the disc. Interpretation of these is a skill to be learned, however, so at this point the student might turn to the 'Skills' option, and work through a tutorial on site plan reading. The tutorial begins with a typical archaeological map, in this case of the site of Mycenae. Here there is a specific task set, to identify specific items on the site by pointing to the section where they are represented. Clues are provided in the form of stills taken from the video of the site itself, with descriptions of the item selected. If the student points to the wrong part of the map for a selected item, they are given further visual and informational hints to help in the identification. Feedback can therefore be more adaptive here than in the case of the open-ended questions. Equipped with the some interpretative skills, the student can now use them in gathering evidence from the site plan of the nature of the society which existed there. Combining this with the notes already made in the Notepad on the basis of the text searches allows the student to assemble a synthesis from different sources in building up their answer to the question, which again can be compared with the model offered in the Discussion.

The final Activity in Week One (see Figure 1) is the fundamental point that the study guide builds to for the week's work. In the printed version this is the denouement: after all the analysis carried out during the week the issue of the extent to which we can say that the poem does illuminate a historical context remains debatable. In the interactive media version, the question draws directly on the outcomes from all the previous activities, each of which has been assembled by contributions from the student. This is the main difference from the print version. By structuring the student's activities it has been possible to maintain the original narrative line, but by involving the student in the development of each part of the story they now have a greater involvement in the concluding analysis, being aware that this was where they were heading, right from the start. The denouement is not so much the rabbit pulled out of the hat, but a conclusion they have played some part in building. This is not impossible to achieve in the print version, of course. A teaching text will attempt to clarify the argument at each stage, involve the student in seeing how each stage follows, and the good student will critique and question and do their own analysis and interpretation as they work through the guide. But the interactive media version requires more action by the student, and provides the tools to do it with, and by exposing the narrative line from the start brings them in as participant in the development of the story, rather than recipient of it.

To summarise, the design features built into the Homer disc worked to the following general guidelines:

- make the narrative explicit as a series of sub-goals

- make the learner active in achieving goals - search resources making notes

- give feedback to allow amendment - discussion and amended note

- build to a integrative goal

In doing so, we have not yet solved the research question set out above as 'finding the investigate techniques students need in order to conduct resource-based learning. We have met Meno's paradox by prompting the questions the student must ask of the resource, and enabling them to 
know how well they have answered them by providing access to model answers. The system itself cannot evaluate answers that go beyond what the model answer provides, but at least it stimulates the possibility that such answers are constructed, which can then be built into the student's assignment to be assessed by the human tutor.

\section{Evaluation}

The extent to which the current design is a successful learning experience was evaluated qualitatively during its first implementation with students who had previously studied the course in its traditional form. We wanted a sense of the contrast between the two forms, and it is not feasible to do a quantitative comparison through a control group study with two groups of students, given the expense of equipping a large number of students in their homes with multimedia PCs. In each run of the evaluation we delivered PCs to two students in their homes for a 6-week period. Each student was studied by an evaluator visiting them for a two-hour session of observation plus interview, and was interviewed by telephone approximately every two weeks.

Comments from students using the system in this way generated several key findings.

\section{(i) Retain the mix of media}

Although the design makes the overall narrative line explicit in the sub-goals for the activities each week, it is clear that students still derive their sense of structure from the print:

"... But it does seem bitty. The flow comes from the learning guides. This is all sound-bites and photo-calls."

"I would not read off the screen. I want books as well, but I would like to use it as a replacement for Learning Guide 2, along with the other material."

"I felt I was being ordered around. It was very directive. But it's quite structured and supportive. It could induce panic without that."

The final comment demonstrates the delicate balance that must be made between giving enough sense of narrative and structure to avoid panic and confusion, without over-directing people. In fact the interface design allows the learner to anything at any time, while providing a default path - even so, the sense of control was palpable for this student.

\section{(ii) The design promotes active learning}

The design was more successful in making the students active learners. The hypertext links between the poems and the commentaries, for example, increase the likelihood of students pursuing those links:

"I did find it easier to click on the Commentary. It's far superior to books in that way."

"I like it as a way of working very much, particularly the hypertext links, being able to do that easily." 
"I have done a lot more on the Companion, because it is easy to do - just click without interrupting the flow, - but getting there is more difficult in print so I don't do it."

The principal analytical device available to students in the multimedia that is genuinely different from the print version is the dynamic search facility within Toolbook. This account from a student illuminates why and how this makes such a difference to what and how he learns:

"The search facility is very exciting.... if I [had] had the text searchable like this I could have reached a greater level of understanding more quickly... I realise that the process of searching is part of the learning process, but sometimes it interrupts the flow of what you wish to write."

The problem of 'interrupting the flow' is one that all academics and scholars will recognise, and one sympathises with the student who has a high cognitive overload to manage, in the form of grappling with difficult ideas, constructing arguments, marshalling evidence, articulating their own analysis - it is a lot to keep track of, and a computer-based tool that streamlines just one part of it will be highly valued.

\section{(iii) The Notepad provides a valuable activity}

One of our anxieties was that students would not make good use of the Notebook. This was important, because it was our only means of creating some kind of feedback - to encourage the students to commit themselves to their own ideas before being given access to the 'model answer'. If they treated the Notebook with the same neglect they did the space in the printed text where they were supposed to write something before reading on, then its value would be lost. So far, however, the evaluation suggests that the new medium affords a sufficiently different response that students do use the Notebook as planned:

"The first week I did 4-5 pages [in the Notebook], also the second week."

"I didn't go back to change [notes after seeing the Discussion] ... but I like it, I do have a tendency to be a bit superior and tend to skip the activities. Being forced to do it - I like that."

Actually, they are not forced. It would be possible to type nonsense into the Notebook and still be given access, but this would probably be seen as being a little too much like cheating - there is just enough sense of coercion in the program to encourage the students to commit themselves first, as we hoped.

\section{(iv) The value of explicit testing is ambiguous}

Where we could ask explicit questions with specific answers that could be checked, we saw this as preferable, because it would have the advantage of changing students' own self-perceptions:

"It gave me a few surprises. It took me three attempts to get the right characters [in one of the tasks set]. I felt I knew it better than that."

One student, however, could see a disadvantage:

"If you perform the tasks, you might feel you'd done it, you'd studied it. Books leave you with some sense of more to do." 
This is a sophisticated insight into the dependency that any assessment system can induce in students. While the knowledge remains to be explored you know you have more to do. As soon as you are tested and pass there must be a sense of mastery, which is always unwarranted, given the selective nature of assessment. Although this is a universal problem in education, it must give us pause, as we design stand-alone teaching programs, if we are to avoid inducing instrumentalist approaches by students with closed questions and quizzes.

\section{(v) The design encouraged high level processing of the material}

Although the narrative was decomposed into its constituent parts for the multimedia activities, we still had to retain the final overarching activity as the overall question concerning the link between the two sources of data, the poem and the archaeology.

Enabling students to take part in building towards this integrative goal, rather than be led towards it, inexorably, in the print, meant that they could experience the content in a different way:

"Many OU courses are in danger, even at third level, of channelling you towards certain beliefs."

"I wish I'd had it before" - "Why?" - "The ability to search the text, there's no other way to pick up certain things. You can be like a scholar."

This is what we are aiming for. If we can really use the medium to create the constructivist's environment in which the learner can explore, in a supported way, what it means to be a scholar in that field, then we shall really have achieved the educational potential these media offer. For these students it worked, but they are a very small sample so far.

Further evaluation is now being carried out with the students who are studying the course for the first time, on a larger scale. This will give us additional insights into how students distribute their workload among the different media, and how they differentiate the learning outcomes afforded by each one. The task of the research project now is to investigate whether the early evaluation results persist, whether they transfer to other multimedia programs, whether students do contribute more of their own thinking, and can make sense of the activities undertaken in the context of an overall narrative line. Then the task is to investigate how far this kind of scaffolding can be taken away? Is there a generalisable form of questioning and activity-setting applicable to a wider range of resource discs? How much help does Meno's slave need to conduct the dialogue with Socrates?

\section{Concluding Points}

The paradox of interactive media is that being a user-control medium the learner expects to have control, and yet a learner does not know enough to be given full control. The role of researcher using a multimedia database is wonderful for the expert scholar, but not for the learner. Finding their way for the first time through this subject area, novices cannot be expected to set appropriate goals, or plot a reasonable path, they will under-specify the problem, be distracted by irrelevancies, be unsure how to evaluate the information they find, over-generalise from instances, remain unaware of incompleteness, fail to recognise inconsistencies... there is every opportunity to fail to meet the objective. This kind of multimedia can only be relied upon to achieve the desired objectives if it is used together with extensive teacher input in the form of preparation, supervision and de-briefing, in order to put back the guidance and support that is missing. Most learners are not yet ready to be researchers. There is still a place for narrative. 


\section{References}

1. Plowman, L. An Investigation of Design Issues for Group Use of Interactive Video. Ph.D. thesis, Brighton Polytechnic. (1991).

2. Stratfold, Matthew Investigation into the Design of Educational Multimedia: Video, Interactivity and Narrative. Unpublished PhD thesis, Open University. (1994)

3. Laurillard, D Rethinking University Teaching: A Framework for the Effective Use of Educational Technology. London: Routledge. (1993).

4. Mandler, J. M. and deForest, M. Is there more than one way to recall a story? Child Development, 50, 886-889, (1979).

5. Plowman, L. An ethnographic approach to analysing navigation and task structure in interactive multimedia: some design issues for group use. In A. Monk, D. Diaper, M. D. Harrison (eds). People and Computers VII, (271-287). Cambridge University Press: Cambridge. (1992).

6. Laurillard D, Baric L, Chambers P, Easting G, Kirkwood A, Plowman L, Russell P, Taylor J Interactive Media in the Classroom: Report of the Evaluation Study. National Council of Educational Technology: Coventry. (1994)

\section{Acknowledgements}

The course 'Homer: Poetry and Society' was created by the A295 course team, chaired by Dr Chris Emlyn-Jones.

The 'Homer CD' was designed and created by the project team chaired by Dr Joel Greenberg.

The project 'MENO: Multimedia, Education and Narrative Organisation' is funded for three years ( $£ 230,000$ for 1995-1998) within the Cognitive Engineering Initiative of the Economic and Social Research Council, UK. Collaborating researchers are Prof Diana Laurillard (Open University), Dr Rose Luckin (University of Sussex), Dr Lydia Plowman (Scottish Council for Educational Research), Dr Matthew Stratfold and Dr Josie Taylor (Open University).

\section{List of figures}

Figure 1: An example of the set of investigation activities for a week's work.

Figure 2: Advice offered to students for Activity 1.

Figure 3: The result of a search conducted for Activity 1

Figure 4: The expert's 'Discussion' associated with Activity 1. 